\title{
18F-Flumazenil: A $\gamma$-Aminobutyric Acid A-Specific PET Radiotracer for the Localization of Drug-Resistant Temporal Lobe Epilepsy
}

\author{
Lucy Vivash ${ }^{1}$, Marie-Claude Gregoire ${ }^{2}$, Eddie W. Lau ${ }^{3}$, Robert E. Ware ${ }^{3}$, David Binns ${ }^{3}$, Peter Roselt ${ }^{3}$, \\ Viviane Bouilleret ${ }^{1}$, Damian E. Myers ${ }^{1}$, Mark J. Cook ${ }^{4}$, Rodney J. Hicks ${ }^{3}$, and Terence J. O’Brien ${ }^{1}$ \\ ${ }^{I}$ Departments of Medicine and Neurology, Melbourne Brain Centre, The Royal Melbourne Hospital, University of Melbourne, Royal \\ Parade, Parkville, Victoria, Australia; ${ }^{2}$ ANSTO LifeSciences, Australian Nuclear Science and Technology Organisation, Menai, \\ Sydney, New South Wales, Australia; ${ }^{3}$ The Centre for Molecular Imaging, Peter MacCallum Cancer Centre, East Melbourne, Victoria, \\ Australia; and ${ }^{4}$ The Departments of Medicine and Neurology, St. Vincent's Hospital, The University of Melbourne, East Melbourne, \\ Victoria, Australia
}

Studies report that ${ }^{11} \mathrm{C}$-flumazenil (FMZ) PET more specifically localizes the epileptogenic zone in patients with medically refractory focal epilepsy than ${ }^{18} \mathrm{~F}-\mathrm{FDG}$ PET. However, practical aspects of ${ }^{11} \mathrm{C}$ use limit clinical application. We report a phase $\mathrm{I} / \mathrm{ll}$ a study assessing the clinical use of ${ }^{18} \mathrm{~F}-\mathrm{FMZ}$ PET for the localization of the epileptogenic zone in patients with drug-resistant temporal lobe epilepsy (TLE). Receptor binding was quantified using kinetic modeling that did not require arterial sampling. Methods: Dynamic ${ }^{18} \mathrm{~F}-\mathrm{FMZ}$ PET and static interictal ${ }^{18} \mathrm{~F}-\mathrm{FDG}$ PET scans were compared in healthy controls ( $n=17$ for ${ }^{18} \mathrm{~F}-\mathrm{FMZ}$ and $n=20$ for ${ }^{18} \mathrm{~F}-\mathrm{FDG}$ ) and TLE patients with mesial temporal sclerosis on MR imaging (MTS, $n=12$ ) and with normal MR imaging (NL TLE, $n=19$ ). Masked visual assessment of images was undertaken. Parametric images of ${ }^{18} \mathrm{~F}-$ $\mathrm{FMZ}$ binding potential $\left(\mathrm{BP}_{\mathrm{ND}}\right)$ were generated using the simplified reference tissue model. Region-of-interest analysis on coregistered MR images and statistical parametric mapping were used to quantify ${ }^{18} \mathrm{~F}-\mathrm{FMZ} \mathrm{BP}_{\mathrm{ND}}$ and ${ }^{18} \mathrm{~F}-\mathrm{FDG}$ uptake in the temporal lobe. Results: The visual assessment of static standardized uptake value images showed ${ }^{18} \mathrm{~F}-\mathrm{FMZ}$ PET to have high specificity (16/17 [94\%]) and moderate sensitivity (21/31 [68\%]) for the localization of the epileptogenic zone, with a more restricted abnormality than ${ }^{18} \mathrm{~F}-$ FDG PET. However, the ${ }^{18} \mathrm{~F}-\mathrm{FMZ}$ standardized uptake value images were falsely localizing in 3 of 31 patients (10\%). Region-of-interest analysis demonstrated reductions in ipsilateral hippocampal ${ }^{18} \mathrm{~F}-$ $\mathrm{FMZ} \mathrm{BP}_{\mathrm{ND}}$ in patients with either MTS or NL TLE, compared with controls subjects. Ipsilateral hippocampal ${ }^{18} \mathrm{~F}-\mathrm{FMZ} \mathrm{BP}_{\mathrm{ND}}$ was independent of both hippocampal volume and ${ }^{18} \mathrm{~F}-\mathrm{FDG}$ uptake, whereas ipsilateral hippocampal volume was correlated with ${ }^{18} \mathrm{~F}-\mathrm{FDG}$ uptake $\left(r^{2}=0.69, P<0.0001\right)$. Statistical parametric mapping analysis demonstrated decreased uptake in 14 of $31(45 \%)$ cases with ${ }^{18} \mathrm{~F}-\mathrm{FMZ}$ PET and 18 of 29 (62\%) with ${ }^{18}$ F-FDG PET. Cluster size was significantly smaller on ${ }^{18} \mathrm{~F}-\mathrm{FMZ}$ than ${ }^{18} \mathrm{~F}-\mathrm{FDG}$ images (37 vs. 160 voxels, $P<0.01$ ). Conclusion: ${ }^{18} \mathrm{~F}-\mathrm{FMZ}$ PET has potential as a clinical tool for the localization of the epileptogenic zone in the presurgical evaluation of drug-resistant TLE, providing information complementary to ${ }^{18} \mathrm{~F}-\mathrm{FDG} \mathrm{PET}$, with a more restricted region of abnormality.

Received Apr. 16, 2012; revision accepted Mar. 20, 2013.

For correspondence or reprints contact: Terence J. O'Brien, Department of Medicine, The Royal Melbourne Hospital, Royal Parade, Parkville, 3050, Victoria, Australia.

E-mail: obrientj@unimelb.edu.au

Published online Jul. 15, 2013.

COPYRIGHT (C 2013 by the Society of Nuclear Medicine and Molecular Imaging, Inc.
Key Words: ${ }^{18} \mathrm{~F}$-flumazenil; positron emission tomography; FDG; temporal lobe epilepsy; fluorodeoxyglucose

J Nucl Med 2013; 54:1270-1277

DOI: 10.2967/jnumed.112.107359

$\mathbf{T}$ emporal lobe epilepsy (TLE) is the most common form of drug-resistant epilepsy in adults. In patients with drug-resistant TLE, surgery is frequently the only therapeutic option with a realistic chance of rendering the patient seizure-free. One of the major goals of the presurgical evaluation is the identification of the site and extent of the epileptogenic zone. This is usually achieved by the concordance of complementary methods, in particular MR imaging and video-electroencephalographic monitoring, commonly supplemented by ${ }^{18}$ F-FDG PET or ictal SPECT (1). However, the area of abnormal metabolism identified by interictal ${ }^{18} \mathrm{~F}$-FDG PET, or of hyperperfusion on ictal SPECT, often extends well beyond the epileptogenic zone across a large portion of the temporal lobe (and surrounding regions) $(2,3)$. Furthermore, the localization rate of ${ }^{18} \mathrm{~F}$-FDG PET in patients with extratemporal epilepsy is low (4), and obtaining true ictal SPECT injections is resource-intensive and logistically difficult for many centers. Therefore, the development and validation of new PET radiotracers that may more accurately identify the epileptogenic zone would be of great clinical value.

Decreased $\gamma$-aminobutyric acid A/central benzodiazepine receptor $\left(\mathrm{GABA}_{\mathrm{A}} / \mathrm{cBZR}\right)$ density in association with the epileptogenic zone has been well described (5). Studies have reported that PET imaging using the $\mathrm{GABA}_{\mathrm{A}} / \mathrm{cBZR}$-specific radiotracer ${ }^{11} \mathrm{C}$ flumazenil $\left({ }^{11} \mathrm{C}\right.$-FMZ) identifies a more restricted region of abnormality in the epileptogenic zone and has a higher sensitivity for extratemporal localizations $(6,7)$. However, practical limitations of using ${ }^{11} \mathrm{C}$-in particular, its short 20 -min half-life, necessitating an onsite cyclotron, and the need for arterial blood sampling (8) or multiinjection protocols $(8,9)$ to model the tracer-binding characteristics-have prevented its integration into routine clinical practice. These limitations have motivated the development of ${ }^{18} \mathrm{~F}$-radiolabeled FMZ conjugates for PET imaging $(10,11)$. ${ }^{18} \mathrm{~F}$ has a significantly longer half-life than ${ }^{11} \mathrm{C}$, approximately $110 \mathrm{~min}$, and a superior signal-to-noise ratio, making it more 
practical for routine clinical use (12). We have previously shown in rats that ${ }^{18} \mathrm{~F}-\mathrm{FMZ}$ has suitable characteristics as a PET radiotracer, possessing a favorable metabolic profile and appropriate kinetics for modeling (10).

This study reports the use of ${ }^{18} \mathrm{~F}-\mathrm{FMZ}$ PET in both patients with TLE and a group of well-characterized controls. This phase I/IIa trial aimed to provide proof-of-concept data for the clinical use of ${ }^{18} \mathrm{~F}$ FMZ as a PET radiotracer for the localization of the epileptogenic zone in patients with drug-resistant TLE. The images were analyzed without the need for arterial blood sampling to enable the more practical application of this technology to clinical practice. The findings on ${ }^{18} \mathrm{~F}-\mathrm{FMZ}$ PET were contrasted with those of ${ }^{18} \mathrm{~F}-\mathrm{FDG}$ PET, the current best functional imaging technique for localizing the epileptogenic zone interictally in patients with drug-resistant TLE.

\section{MATERIALS AND METHODS}

\section{Patient Population}

Thirty-one patients with drug-resistant TLE (median age, $39 \mathrm{y}$; age range, 17-72 y; 11 men, 20 women) were studied. The patients were divided into the following 2 subgroups: well-localized unilateral TLE with concordant mesial temporal sclerosis and ${ }^{18} \mathrm{~F}$-FDG hypometabolism (based on routine clinical reports) (MTS, $n=12$ ) and well-localized unilateral TLE with concordant ${ }^{18} \mathrm{~F}-\mathrm{FDG}$ hypometabolism without MTS or other structural lesion (NL, $n=19$ ). All patients underwent at least $5 \mathrm{~d}$ of video-electroencephalographic monitoring to confirm the localization of the epileptogenic zone. For each patient, the determination of the localization of the epileptogenic zone was made at a meeting attended by at least 2 experienced epileptologists based on a concordance of all available clinical, video-electroencephalographic, imaging, and neuropsychologic data. Table 1 details the demographics and clinical features of the 3 participant groups.

Two control groups were studied -17 healthy subjects (median age, 34 y; age range, 23-60 y; 8 men, 9 women) underwent MR imaging and ${ }^{18} \mathrm{~F}-\mathrm{FMZ}$ PET, and a separate cohort of 20 healthy subjects underwent ${ }^{18} \mathrm{~F}-\mathrm{FDG}$ PET (median age, $32 \mathrm{y}$; age range, 20-57 y; 10 men, 10 women). Information on the latter group was previously reported in a comparison with ${ }^{18} \mathrm{~F}$-FDG uptake in epileptic subjects (3).

The study was approved by the Melbourne Health Human Ethics Research Committee (HREC \#2007.286). All subjects provided written informed consent before participating in any study procedures.

\section{PET Imaging}

${ }^{18} \mathrm{~F}-\mathrm{FMZ}$ PET and ${ }^{18} \mathrm{~F}-\mathrm{FDG}$ PET scans were obtained at the Peter MacCallum Cancer Centre on the Discovery STE PET/CT scanner (GE Healthcare). The PET camera has bismuth germanate oxide detectors that acquire 47 contiguous slices with a thickness of $3.27 \mathrm{~mm}$ (axial length, $15.4 \mathrm{~cm}$ ). Images were acquired in 3 dimensions, and the intrinsic axial resolution was $5.1 \mathrm{~mm}$ at a radius of $1 \mathrm{~cm}$ and $5.8 \mathrm{~mm}$ at a radius of $10 \mathrm{~cm}$. The full width at half maximum of the scan was $5.4 \mathrm{~mm}$. All studies were reconstructed into a $35-\mathrm{cm}$ diameter, $128 \times 128$ transaxial matrix using the 3D VUE point (GE Healthcare) ordered-subset expectation maximization algorithm with 8 iterations, 8 subsets, and a gaussian postprocessing filter of $3.82 \mathrm{~mm}$ (full width at half maximum). A lowdose coacquired CT scan was used for attenuation correction.

${ }^{18} \mathrm{~F}-\mathrm{FMZ}$ was prepared according to the method described by Ryzhikov et al. (13). Radiochemical purity was greater than $99 \%$, and specific activity ranged from 125.06 to $480.63 \mathrm{GBq} / \mu \mathrm{mol}$ at the end of synthesis.

Subjects were intravenously injected with ${ }^{18} \mathrm{~F}-\mathrm{FMZ}$ (4.23 MBq/kg; mean, $322 \mathrm{MBq}$; range, 197-481 MBq) via the left antecubital vein, and immediately a dynamic list-mode image of $1 \mathrm{~h}$ was acquired. Data were reframed into $10 \times 30 \mathrm{~s}, 5 \times 1 \mathrm{~min}$, and $10 \times 5 \mathrm{~min}$ frames.
Participants' temperature, blood pressure, and oxygen saturation were monitored before and $90 \mathrm{~min}$ after injection of ${ }^{18} \mathrm{~F}$-FMZ. Participants were also monitored for other signs of adverse effects on-site for 90 min after injection of ${ }^{18} \mathrm{~F}-\mathrm{FMZ}$ and asked to report any ensuing adverse effects. Video monitoring during imaging and a postimaging interview were conducted to ensure patients did not experience a seizure during image acquisition.

Patients also underwent ${ }^{18} \mathrm{~F}$-FDG PET as part of their routine clinical investigation (within $4 \mathrm{y}$ of ${ }^{18} \mathrm{~F}-\mathrm{FMZ}$ scan), as previously described (4). The electronic files for the ${ }^{18} \mathrm{~F}$-FDG PET scans were unavailable for $2 \mathrm{NL}$ patients and therefore were unable to be included in the analysis. All patients were imaged as outpatients in the interictal state. Patients fasted for $4 \mathrm{~h}$ before the scan and rested in a quiet, darkened room for $15 \mathrm{~min}$ before ${ }^{18} \mathrm{~F}$-FDG administration and for at least $30 \mathrm{~min}$ afterward. ${ }^{18} \mathrm{~F}$-FDG $(250 \mathrm{MBq}$ ) was injected intravenously. Scanning commenced $60 \mathrm{~min}$ after radiotracer administration. Patients were scanned for $20 \mathrm{~min}$ in a single bed position, with the head positioned comfortably and immobilized with tape.

${ }^{18} \mathrm{~F}-\mathrm{FDG}$ and FMZ PET/CT data were exported in Digital Imaging and Communications in Medicine P10 format for analysis.

Healthy control ${ }^{18} \mathrm{~F}$-FDG PET images were obtained for a previous study (3).

\section{MR Imaging}

Participants were scanned on a 1.5-T clinical whole-body scanner (Sigma Horizon SE120; GE Healthcare) using a standardized protocol including a T1-weighted sagittal localizer, a coronal T1-weighted wholebrain volumetric series, axial and coronal T2- and fluid-attenuated inversion recovery (FLAIR)-weighted images, an oblique-coronal diffusion-weighted imaging (DWI) sequence, an oblique coronal T2 mapping sequence, and an oblique coronal echo-planar imaging (EPI) sequence. The whole-brain volumetric series was acquired using a fast spoiled gradient-echo technique with the following parameters: slice thickness, 1.5-mm; interslice gap, 0; repetition time/echo time, 11/2 ms pulse sequence; field of view, $22 \times 22 \mathrm{~cm}$; and matrix size, $256 \times 256$.

The criterion for classifying a patient as having unilateral MTS for the study was based on the clinical report by the neuroradiologist. This clinical diagnosis was made using standard visually assessed criteria, including hippocampal atrophy, altered signal (i.e., increased T2, decreased T1), and loss of internal architecture in the hippocampus (14).

\section{Masked Visual Assessment of PET Images}

Two experienced nuclear medicine physicians, masked to clinical data and imaging results, independently assessed all image sets. The first 5-min frame, corresponding to 10-15 min after injection, was used for the assessment of the static ${ }^{18} \mathrm{~F}-\mathrm{FMZ}$ standardized uptake value (SUV) images and the static ${ }^{18} \mathrm{~F}-\mathrm{FDG}$ images used. The image sets were presented in a random order, including both the ${ }^{18} \mathrm{~F}-\mathrm{FMZ}$ and the ${ }^{18} \mathrm{~F}-$ FDG images from patients and controls as well as those from an additional 4 patients with other epilepsy syndromes so that the reviewers could not assume that the abnormal images localized to the temporal lobe. The reviewers were asked to localize the images to 1 of 8 sites (left or right; frontal, temporal, parietal, or occipital) or classify them as nonlocalizing. Reviewers were also asked to specify the level of confidence of their choice (nonlocalizing/lateralizing/probably localizing/ definitely localizing) and to grade the extent and severity of the abnormality (mild to severe). If the 2 reviewers disagreed, a third masked reviewer was used. If this third reviewer classified the image localizing in agreement with 1 of the primary reviewers, the image was considered localizing, otherwise the image was considered nonlocalizing.

The results of the PET scans were assessed for sensitivity, specificity, and Cohen's $\kappa$-scores of interobserver agreement. The extent and severity of the abnormality were quantified by weighting the severity of the abnormality across all regions affected (number of 
TABLE 1

Demographic and Clinical Characteristics of Study Groups

\begin{tabular}{|c|c|c|c|c|}
\hline Variable & $\operatorname{MTS}(n=12)$ & $\mathrm{NL}(n=19)$ & Control $(n=17)$ & $P$ \\
\hline Age $(y)$ & & & & 0.48 \\
\hline Median & 40 & 36 & 29 & \\
\hline Interquartile range & $37.25-48$ & $37.5-45.25$ & $25-44$ & \\
\hline Sex & & & & 0.63 \\
\hline Male & 5 & 6 & 8 & \\
\hline Female & 7 & 13 & 9 & \\
\hline Duration of epilepsy (y) & & & Not applicable & 0.001 \\
\hline Median & 18 & 3 & & \\
\hline Interquartile range & $11.5-24.5$ & $1.88-7.75$ & & \\
\hline Number of antiepileptic drugs & & & Not applicable & 0.15 \\
\hline Median & 3 & 2 & & \\
\hline Interquartile range & $2-3$ & $1-3$ & & \\
\hline Epilepsy lateralization & & & Not applicable & 0.47 \\
\hline Left & 5 & 11 & & \\
\hline Right & 7 & 8 & & \\
\hline Epilepsy surgery & 5 & 5 & Not applicable & 0.45 \\
\hline $\begin{array}{l}\text { Hospital Anxiety and } \\
\text { Depression Score (HADS)* }\end{array}$ & & & & 0.002 \\
\hline Median & $4^{\dagger}$ & $6^{\ddagger}$ & 1 & \\
\hline Interquartile range & $2.5-11$ & $2-8.5$ & $0-1$ & \\
\hline Depression score $10<$ & 3 & 4 & 0 & 0.04 \\
\hline HADS anxiety score* & & & & 0.006 \\
\hline Median & 6 & $8.5^{\ddagger}$ & 3 & \\
\hline Interquartile range & $4-10.25$ & $5-12$ & $2-6$ & \\
\hline Anxiety score $10<$ & 3 & 8 & 0 & 0.03 \\
\hline
\end{tabular}

${ }^{*} n$ for HADS: MTS, $n=8$; NL, $n=18$; controls, $n=12$.

${ }^{\dagger} P<0.05$ MTS vs. control.

${ }^{\ddagger} P<0.01 \mathrm{NL}$ vs. control.

Scores of $10<$ on depression and anxiety branches of HADS questionnaire are indicative of depression/anxiety. Statistics: 1-way ANOVA age/HADS, Fisher exact surgery, lateralization, $\chi^{2}$ sex, Mann-Whitney $U$ antiepileptic drugs, and epilepsy duration.

regions involved multiplied by the severity in that region: mild, 1; moderate, 2; and severe, 3).

\section{Generation of Parametric Images of ${ }^{18} \mathrm{~F}-\mathrm{FMZ}$ Binding Potential $\left(\mathbf{B P}_{\mathrm{ND}}\right)$}

Parametric images of ${ }^{18} \mathrm{~F}-\mathrm{FMZ} \mathrm{BP}_{\mathrm{ND}}$ were generated using PMOD software (PMOD Technologies Ltd.) and the simplified reference tissue model (SRTM) (15). The receptor-rich (left thalamus and left occipital cortex) and reference (pons) regions were delineated on the MR images coregistered to the PET data. Time-activity curves calculated for the 2 regions were used to calculate the set of basic functions.

\section{Masked Visual Assessment of Parametric ${ }^{18} \mathrm{~F}-\mathrm{FMZ}$ BP $_{\text {ND }}$ Images}

The ${ }^{18} \mathrm{~F}-\mathrm{FMZ} \mathrm{BP} \mathrm{ND}_{\mathrm{ND}}$ images were visually assessed in a masked manner, as described above for the static ${ }^{18} \mathrm{~F}$-FMZ SUV images and ${ }^{18} \mathrm{~F}$ FDG images, by the same 3 experienced nuclear medicine physicians.

\section{Region-of-Interest (ROI) Analysis of Parametric ${ }^{18} \mathrm{~F}-\mathrm{FMZ}$ $\mathrm{BP}_{\mathrm{ND}}$ and Static ${ }^{18} \mathrm{~F}-\mathrm{FDG}$ Images}

After the generation of parametric ${ }^{18} \mathrm{~F}-\mathrm{FMZ} \mathrm{BP}_{\mathrm{ND}}$ images, ROI analysis was performed on both static ${ }^{18} \mathrm{~F}-\mathrm{FDG}$ images and parametric ${ }^{18} \mathrm{~F}_{-\mathrm{FMZ}} \mathrm{BP}_{\mathrm{ND}}$ images using Analyze (version 10.0; Mayo Clinic). ROIs for the hippocampi and anterior and posterior temporal lobes were delineated on the MR images manually by a single operator masked to the PET data. The hippocampi were delineated on coronal slices of the MR images, followed by adjustments in the sagittal plane. Anterior and posterior temporal lobes were delineated as previously described (3). These regions were then applied to the ${ }^{18} \mathrm{~F}-\mathrm{FDG}$ and ${ }^{18} \mathrm{~F}-\mathrm{FMZ} \mathrm{BP}_{\mathrm{ND}}$ images, from which ${ }^{18} \mathrm{~F}-\mathrm{FDG}$ uptake and ${ }^{18} \mathrm{~F}-\mathrm{FMZ}$ $\mathrm{BP}_{\mathrm{ND}}$ values were derived. Additionally, the cerebellum was delineated and applied to ${ }^{18} \mathrm{~F}$-FDG images for normalization of ${ }^{18} \mathrm{~F}-\mathrm{FDG}$ uptake values (a second analysis using the pons for normalization of ${ }^{18} \mathrm{~F}-\mathrm{FDG}$ uptake was performed; however, the resultant values were more variable than those derived using the cerebellum) (Supplemental Fig. 3; supplemental materials are available online only at http://jnm.snmjournals.org). These values were compared between hemispheres and against the control population to localize the epileptogenic zone. The ${ }^{18} \mathrm{~F}-\mathrm{FDG}$ PET scans from the controls did not have matching MR images, so these scans were registered to an in-house MR template. However, 5 controls had to be excluded because of poor coregistration with the template MR images.

\section{Statistical Parametric Mapping (SPM) Analysis of ${ }^{18} \mathrm{~F}-\mathrm{FMZ}$ $\mathrm{BP}_{\mathrm{ND}}$ and Static ${ }^{18} \mathrm{~F}-\mathrm{FDG}$ Images}

The PET and SPECT tool in SPM5 (Wellcome Institute of Neurology, UCL) was used. Participants' images were normalized into the standard PET template provided in SPM5 using a 12-parameter affine transformation. Individual patient ${ }^{18} \mathrm{~F}-\mathrm{FMZ} \mathrm{BP}_{\mathrm{ND}}$ and ${ }^{18} \mathrm{~F}-\mathrm{FDG}$ images were compared with the control group for areas of increased and decreased ${ }^{18} \mathrm{~F}-\mathrm{FMZ} \mathrm{BP}_{\mathrm{ND}}$ and ${ }^{18} \mathrm{~F}-\mathrm{FDG}$ uptake. The criteria to consider a cluster of voxels significantly increased or decreased were a $P$ value of less than 0.0005 and voxels greater than 20 , based on previous work (3), a level at which no areas of reduced uptake occurred when individual control images were compared with the remainder of the control group images. The size of the resultant cluster of decreased ${ }^{18} \mathrm{~F}-\mathrm{FMZ} \mathrm{BP}_{\mathrm{ND}}$ and ${ }^{18} \mathrm{~F}-\mathrm{FDG}$ uptake was also measured. 


\section{Statistical Analysis}

Statistical analyses were performed using Statistica (version 5.1; Statsoft Inc.). One-way ANOVA, the Fisher exact test, and the $\chi^{2}$ test were used as appropriate to assess demographics between patient groups. For ROI analysis, the Mann-Whitney $U$ test was used to assess differences between controls and patients. The Wilcoxon rank-sum test was used to assess differences across hemispheres in patients and in cluster sizes after SPM analysis. Pearson correlation was used to assess relationships between variables. Data are expressed as median and interquartile range, and a $P$ value of less than 0.05 was considered significant.

\section{RESULTS}

\section{Tolerability and Adverse Effects}

The ${ }^{18} \mathrm{~F}-\mathrm{FMZ}$ injections were well tolerated, with no subjective or objective adverse effects detected. No seizures were reported by the patients or recorded by the staff during image acquisition.

\section{Masked Review of PET Images}

Figure 1 shows representative static ${ }^{18} \mathrm{~F}$-FDG PET and ${ }^{18} \mathrm{~F}$-FMZ SUV images used for masked visual inspection. On review of the 48 static ${ }^{18} \mathrm{~F}-\mathrm{FMZ}$ images, there was agreement between the 2 primary reviewers for 37 images $(77.1 \%)$, resulting in 11 images requiring assessment by the third reviewer. Interobserver agreement between the 2 primary reviewers was modest at a $\kappa$ of 0.42 . The third reviewer agreed with 1 of the primary reviewers in all but 6 cases. The images were classified as being localizing in 21 of 31 patients and 1 of 17 control subjects, resulting in a sensitivity of $67.7 \%$ and specificity of $94.1 \%$; however, these results included 3 patients in the NL TLE group who were incorrectly localized.

On review of the forty-eight ${ }^{18} \mathrm{~F}-\mathrm{FMZ} \mathrm{BP}_{\mathrm{ND}}$ images, there was agreement between the 2 primary reviewers for 26 images (54.2\%), resulting in 22 images requiring assessment by the third reviewer. Interobserver agreement between the 2 primary reviewers was low at a $\kappa$ of 0.12 . The third reviewer agreed with 1 of the primary reviewers in all but 4 cases. Correct localization of the epileptogenic zone occurred in 16 of 31 patients and all 17 images from the control subjects were classified as nonlocalizing, resulting in a sensitivity of $51.6 \%$ and specificity of $100 \%$.

On review of the forty-nine ${ }^{18} \mathrm{~F}-\mathrm{FDG}$ PET images, there was agreement between the 2 primary reviewers for 38 images (77.6\%). Interobserver agreement between the 2 primary reviewers was modest at a $\kappa$ of 0.60 . The third reviewer agreed with 1 of the primary reviewers in all but 3 cases. Correct localization of the epileptogenic zone occurred in 21 of 29 patients, with all 20

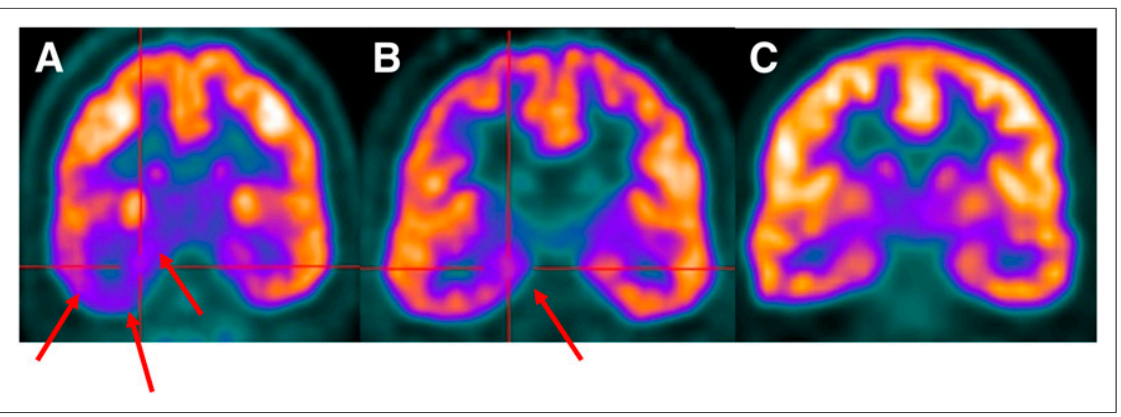

FIGURE 1. Static ${ }^{18} \mathrm{~F}-\mathrm{FMZ}$ PET and ${ }^{18} \mathrm{~F}-\mathrm{FDG}$ PET coronal images used for masked visual review. (A) ${ }^{18} \mathrm{~F}-\mathrm{FDG}$ PET image shows extensive hypometabolism throughout right temporal lobe (arrows). (B) ${ }^{18} \mathrm{~F}-\mathrm{FMZ}$ PET image shows more restricted localization to mesial temporal region in same patient (arrows). (C) Symmetric ${ }^{18} \mathrm{~F}-\mathrm{FMZ}$ distribution in control subject. control images being classified as nonlocalizing, resulting in a sensitivity of $72.4 \%$ and specificity of $100 \%$.

Of the patients with MTS, 10 of $12(83.3 \%)$ were correctly localized on the ${ }^{18} \mathrm{~F}$-FMZ SUV images, compared with 8 of 19 $(42.1 \%)$ for the NL group ( $P=0.03$, Fisher exact test, Table 2$)$, with ${ }^{18} \mathrm{~F}-\mathrm{FMZ} \mathrm{BP} \mathrm{BD}_{\mathrm{ND}}$ images producing similar results, localizing 8 of $12(66.6 \%)$ and 8 of 19 patients $(42.1 \%)$, respectively $(P=$ 0.27 , Fisher exact test). On the ${ }^{18}$ F-FDG PET images, 11 of 12 MTS and 10 of 17 NL patients were correctly localized $(P=0.09$, Fisher exact test). The differences in the localization rate were not significantly different between image sets $\left({ }^{18} \mathrm{~F}-\mathrm{FMZ}\right.$ SUV and ${ }^{18} \mathrm{~F}$ FDG, $P=0.29 ;{ }^{18} \mathrm{~F}-\mathrm{FMZ}$ SUV and ${ }^{18} \mathrm{~F}-\mathrm{FMZ} \mathrm{BP}_{\mathrm{ND}}, P=0.80$; ${ }^{18} \mathrm{~F}-\mathrm{FMZ} \mathrm{BP}_{\mathrm{ND}}$ and ${ }^{18} \mathrm{~F}-\mathrm{FDG}, P=0.12$; Fisher exact test).

The extent of decreased uptake was judged to be more restricted to the mesial temporal region on the ${ }^{18} \mathrm{~F}-\mathrm{FMZ}$ PET images (median severity score, 1.5 ; range, $0-6$ ) than on the ${ }^{18}$ F-FDG PET images (median severity score, 3 ; range, $0.5-12 ; P=0.007$; data not shown) (Figs. 1A and 1B).

\section{ROI Analysis}

Figure 2 shows the results of the ROI analyses of the parametric ${ }^{18} \mathrm{~F}-\mathrm{FMZ}$ PET $\mathrm{BP}_{\mathrm{ND}}$ and static ${ }^{18} \mathrm{~F}$-FDG PET images (normalized to cerebellar uptake) and also the hippocampal volumes. Significant reductions were seen in ${ }^{18} \mathrm{~F}-\mathrm{FMZ} \mathrm{BP}_{\mathrm{ND}}$ in the ipsilateral hippocampus in both patient groups when compared with both the controls and the contralateral hippocampus (Fig. 2A). On MR imaging, both the ipsilateral and the contralateral hippocampal volumes were significantly less in MTS and NL patients than in controls (Fig. 2B). In the MTS group, the ipsilateral hippocampi were significantly smaller than the contralateral hippocampi, whereas in the NL group the magnitude of the volume reductions was similar for both hippocampi. The normalized ${ }^{18}$ F-FDG PET uptake in the ipsilateral and contralateral hippocampal regions was significantly less in both patient groups than in controls (Fig. 2C). In the MTS patients, the relative ${ }^{18}$ F-FDG PET intensity was lower in the ipsilateral than in the contralateral hippocampal region but not in the NL group, where the ${ }^{18}$ F-FDG uptake was symmetrically reduced in both hippocampal ROIs.

Supplemental Figure 1 shows ${ }^{18} \mathrm{~F}-\mathrm{FMZ} \mathrm{BP}_{\mathrm{ND}}$ and ${ }^{18} \mathrm{~F}-\mathrm{FDG}$ PET uptake for the anterior and posterior temporal lobe ROIs. There were no significant differences in ${ }^{18} \mathrm{~F}-\mathrm{FMZ} \mathrm{BP}_{\mathrm{ND}}$ in the anterior temporal lobe between any of the participant groups. A trend to significance was observed in the ipsilateral anterior temporal lobe ${ }^{18} \mathrm{~F}-\mathrm{FMZ} \mathrm{BP} \mathrm{BD}_{\mathrm{ND}}$ between MTS patients and controls (2.39 vs. 3.06, $P=0.06$; Supplemental Fig. 1A). In contrast, ${ }^{18} \mathrm{~F}-\mathrm{FDG}$ PET uptake was significantly reduced in both patient groups, compared with controls in the anterior temporal pole (Supplemental Fig. 1B). This reduction was significantly more marked in the ipsilateral anterior temporal pole in the MTS group but was symmetric in the NL group. The volumes for the anterior temporal lobe ROIs based on the MR imaging did not differ significantly between patients and controls or between hemispheres in patients (data not shown).

There were no significant differences in ${ }^{18} \mathrm{~F}-\mathrm{FMZ} \mathrm{BP}_{\mathrm{ND}}$ in the posterior temporal lobe ROIs (Supplemental Fig. 1C). There was no overall difference in the ${ }^{18} \mathrm{~F}-\mathrm{FDG}$ $\mathrm{PET}$ intensities in the posterior temporal 
TABLE 2

Results of Masked Visual Review of Static ${ }^{18} \mathrm{~F}-\mathrm{FDG}$ and ${ }^{18} \mathrm{~F}-\mathrm{FMZ}$ SUV and $\mathrm{BP}_{\mathrm{ND}}$ Images

\begin{tabular}{|c|c|c|c|c|}
\hline Test & Outcome & MTS & NL TLE & Control \\
\hline \multirow[t]{3}{*}{${ }^{18} \mathrm{~F}-\mathrm{FDG}$} & Localization correct & $11 / 12(91.7)$ & $10 / 17(58.8)$ & $0 / 20(0)$ \\
\hline & Localization incorrect & 0/12 (0) & 0/17 (0) & 0/20 (0) \\
\hline & Not localized & $1 / 12(8.3)$ & 7/17 (41.2) & $20 / 20(100)$ \\
\hline \multirow[t]{3}{*}{${ }^{18} \mathrm{~F}-\mathrm{FMZ}$ SUV } & Localization correct & 10/12 (83.3) & $8 / 19(42.1)$ & $0 / 17(0)$ \\
\hline & Localization incorrect & 0/12 (0) & 3/19 (15.8) & $1 / 17(5.9)$ \\
\hline & Not localized & 2/12 (16.7) & $8 / 19(42.1)$ & $16 / 17(94.1)$ \\
\hline \multirow[t]{3}{*}{${ }^{18} \mathrm{~F}-\mathrm{FMZ} \mathrm{BP}_{\mathrm{ND}}$} & Localization correct & $8 / 12(66.6)$ & $8 / 19(42.1)$ & $0 / 17(0)$ \\
\hline & Localization incorrect & $0 / 12(0)$ & $0 / 19(0)$ & $0 / 17(0)$ \\
\hline & Not localized & 4/12 (33.3) & $11 / 19$ (57.9) & $17 / 17(100)$ \\
\hline
\end{tabular}

Data in parentheses are percentages.

lobe ROIs between the patients and controls; however, there was an asymmetry in the MTS patients, being decreased on the ipsilateral side (Supplemental Fig. 1D). No significant differences were observed in posterior temporal lobe volume between groups or between hemispheres in patients (data not shown).

Supplemental Figure 2 shows ${ }^{18} \mathrm{~F}-\mathrm{FMZ} \mathrm{BP}_{\mathrm{ND}}$ in the hippocampi and anterior and posterior temporal lobes, derived using the occipital cortex as the receptor-rich reference region. Direct comparison of hippocampal ${ }^{18} \mathrm{~F}-\mathrm{FMZ} \mathrm{BP}_{\mathrm{ND}}$ values derived using the 2 reference regions showed no significant difference between the 2 techniques (Supplemental Fig. 2D, $P>0.05$ ).

Supplemental Figure 3 shows ROI analysis of ${ }^{18} \mathrm{~F}-\mathrm{FDG}$ uptake normalized to the pons. The asymmetry between the ipsilateral and contralateral hippocampi in the MTS group is maintained, but patient groups are no longer significantly different from the control values (because of greater variability in the control group) (Supplemental Fig. 3A). Direct comparison of hippocampal ${ }^{18} \mathrm{~F}-$ FDG uptake using the cerebellum and pons for normalization showed no significant difference in the patient groups but a significant difference in the controls.

\section{Correlation Analyses}

Figure 3 shows correlation analyses for the ${ }^{18} \mathrm{~F}-\mathrm{FMZ} \mathrm{BP} \mathrm{ND}_{\mathrm{ND}},{ }^{18} \mathrm{~F}-$ FDG PET, and MR images in the ipsilateral hippocampi of patients. Hippocampal ${ }^{18} \mathrm{~F}-\mathrm{FMZ} \mathrm{BP}_{\mathrm{ND}}$ was not correlated with hippocampal volume $\left(r^{2}=0.15, P=0.44\right.$, Fig. $\left.3 \mathrm{~A}\right)$ or ${ }^{18} \mathrm{~F}$-FDG uptake $\left(r^{2}=\right.$ $0.19, P=0.33$, Fig. 3B). However, a significant correlation was observed between hippocampal ${ }^{18} \mathrm{~F}$-FDG uptake and volume $\left(r^{2}=0.69, P<0.0001\right.$, Fig. 3C).

Figure 4 shows correlation analyses between the duration of the epilepsy and imaging measurements in the ipsilateral hippocampal ROI. No significant correlations were found between hippocampal ${ }^{18} \mathrm{~F}-\mathrm{FMZ} \quad \mathrm{BP}_{\mathrm{ND}}$ and epilepsy duration (Fig. 4A). However, ipsilateral hippocampal volume was found to correlate with epilepsy duration $\left(r^{2}=-0.57, P=0.002\right.$, Fig. 4B) as was ipsilateral hippocampal ${ }^{18} \mathrm{~F}$-FDG uptake $\left(r^{2}=-0.56, P=0.003\right.$, Fig. 4C). No correlation was observed between ${ }^{18} \mathrm{~F}-\mathrm{FMZ} \mathrm{BP}_{\mathrm{ND}}$ and time since last seizure (data not shown), in contrast to the findings of a previous study (16).

MTS ipsilateral $(n=12)$

$\triangle$ MTS contralateral $(n=12)$

- NL ipsilateral $(n=19)$

- NL contralateral $(n=19)$

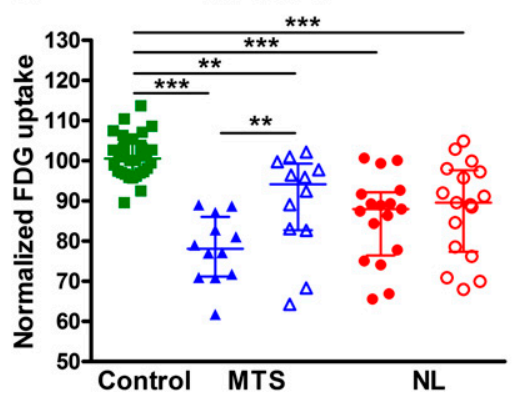

\section{SPM Analyses}

SPM analyses were performed for both parametric ${ }^{18} \mathrm{~F}-\mathrm{FMZ} \mathrm{BP}_{\mathrm{ND}}$ and ${ }^{18} \mathrm{~F}$-FDG PET images for all patients (Fig. 5; Supplemental Fig. 4). SPM analysis showed a cluster of voxels with reduced ${ }^{18} \mathrm{~F}-\mathrm{FMZ} \mathrm{BP}_{\mathrm{ND}}$ in the region of the epileptogenic temporal lobe in 6 of $12(50 \%)$ of MTS and 8 of $19(42 \%)$ of NL patients $(P=1.00$, Fisher exact test). SPM analysis of the ${ }^{18}$ F-FDG PET images showed a cluster of voxels with reduced metabolism in the epilepto- 

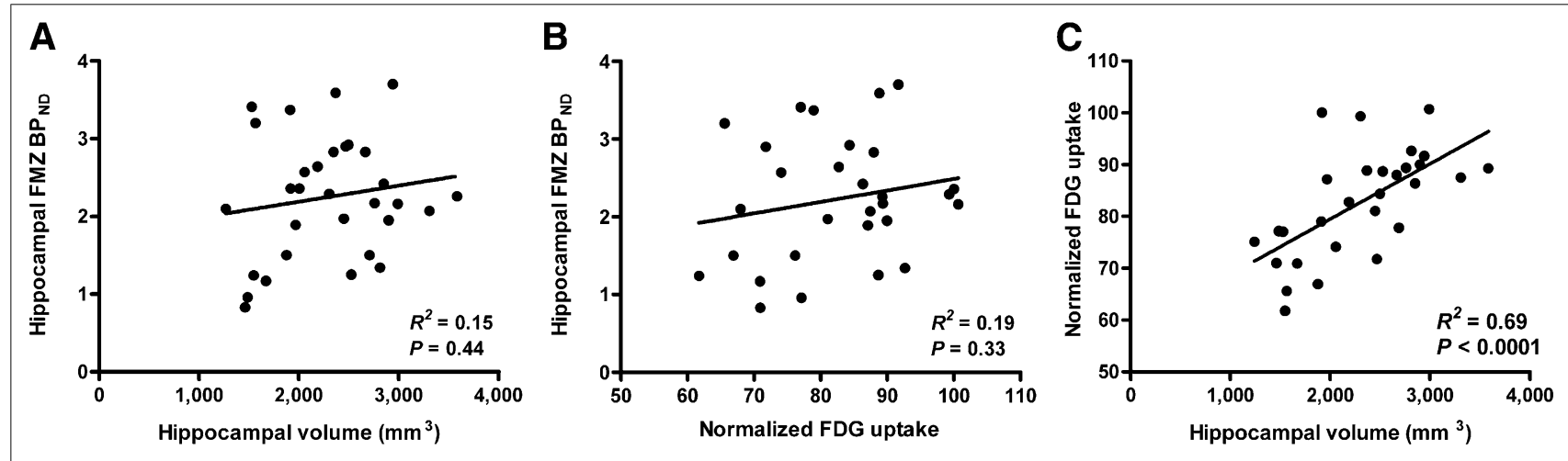

FIGURE 3. Correlation between ${ }^{18} \mathrm{~F}-\mathrm{FMZ} \mathrm{BP}_{\mathrm{ND}}$, normalized ${ }^{18} \mathrm{~F}-\mathrm{FDG}$ uptake, and $\mathrm{MR}$ imaging volumes in ipsilateral hippocampal ROI of patients. ${ }^{18} \mathrm{~F}-\mathrm{FMZ} B \mathrm{BP}_{\mathrm{ND}}$ was independent of both volume $(\mathrm{A})$ and ${ }^{18} \mathrm{~F}-\mathrm{FDG}$ uptake $(\mathrm{B})$; however, ${ }^{18} \mathrm{~F}-\mathrm{FDG}$ uptake and volume were highly correlated $(\mathrm{C})$.

genic temporal lobe in 10 of $12(83 \%)$ of MTS and 8 of $17(47 \%)$ of NL patients $(P=0.06$, Fisher exact test). The cluster size in the epileptogenic temporal lobe was significantly smaller for the ${ }^{18} \mathrm{~F}$ FMZ $\mathrm{BP}_{\mathrm{ND}}$ than the ${ }^{18} \mathrm{~F}-\mathrm{FDG}$ images (Fig. 5A, $n=11, P=$ 0.002 ), indicating a more topographically restricted region of abnormality. Consistent with this, SPM analyses of the ${ }^{18} \mathrm{~F}-\mathrm{FDG}$ PET images showed a greater number of significant clusters in the posterior temporal lobe $(P<0.01)$ and a trend to more clusters in total (Fig. 5B, $P=0.08$ ).

\section{Surgical Outcome}

Eleven patients underwent resective surgery, 6 from the MTS group and 5 from the NL group. In all cases, the histopathologic examination of the resected hippocampus confirmed the preoperative diagnosis of the presence or absence of MTS diagnosed on the basis of the MR imaging, defined by marked loss of neurons in CA1 and CA3 subfields and hilus with relative sparing of the CA2 and dentate regions (17). Five of the patients were seizurefree at the time of last follow-up (median follow-up, $11.5 \mathrm{mo}$; range, 2-25 mo). In the MTS group, 3 of 6 patients were seizurefree after surgery, compared with 2 of 5 in the NL group $(P=1.00$, Fisher exact test). FMZ $\mathrm{BP}_{\mathrm{ND}},{ }^{18} \mathrm{~F}-\mathrm{FDG}$ uptake, volume ROI measures, or results of the SPM analysis were not predictive of surgical outcome (data not shown).

\section{DISCUSSION}

In a control population and TLE patients, this study assessed the use of ${ }^{18} \mathrm{~F}$-FMZ PET in comparison to ${ }^{18} \mathrm{~F}$-FDG for localizing an epileptogenic zone evaluating visual and quantitative analysis of imaging data, including SPM. The primary aim of the study was to generate proof-of-concept data regarding the use of this radiotracer for this purpose, establish that it produced high-quality PET image data and the feasibility of creating parametric $\mathrm{BP}_{\mathrm{ND}}$ maps from the dynamic acquisition data using noninvasive modeling, and confirm safety and tolerability. The results of the ${ }^{18} \mathrm{~F}-\mathrm{FMZ}$ imaging were compared with the current standard PET radiotracer for epilepsy evaluations, ${ }^{18} \mathrm{~F}$-FDG. The study was not powered to demonstrate superiority or inferiority, compared with ${ }^{18}$ F-FDG PET, but rather provide a frame of reference that could be used to design subsequent larger comparative trials.

The study demonstrated that ${ }^{18} \mathrm{~F}-\mathrm{FMZ}$ PET, analyzed without arterial blood sampling, provided high-quality images that specifically localized the epileptogenic zone in most MTS and NL TLE patients studied. The ${ }^{18} \mathrm{~F}-\mathrm{FMZ} \mathrm{BP}_{\mathrm{ND}}$ decrease in the ipsilateral hippocampus was shown to be independent of reductions in hippocampal volume and ${ }^{18} \mathrm{~F}$-FDG uptake. This finding agrees with several previous studies, which have shown that various ${ }^{18} \mathrm{~F}-\mathrm{FMZ}$ binding parameters are independent of hippocampal volume $(7,18,19)$.

In this study, we used a noninvasive method of kinetic modeling to generate parametric images of ${ }^{18} \mathrm{~F}-\mathrm{FMZ} \mathrm{BP}_{\mathrm{ND}}$ - the SRTM (15). This methodology is robust (20), relatively simple to apply, and suitable for use in routine clinical practice. The use of the SRTM is further supported by a previous work by Odano et al. (12), which directly compared ${ }^{18} \mathrm{~F}-\mathrm{FMZ}$ and ${ }^{11} \mathrm{C}-\mathrm{FMZ}$ in healthy volunteers. They showed no difference in uptake kinetics, metabo-
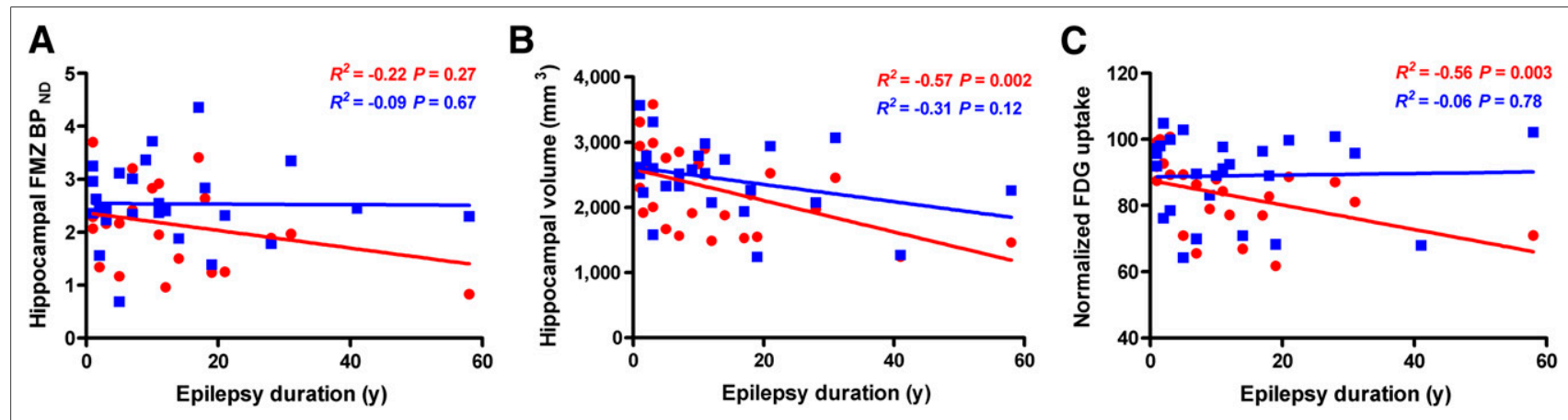

FIGURE 4. Correlation between epilepsy duration and ${ }^{18} \mathrm{~F}-\mathrm{FMZ} \mathrm{BP}_{\mathrm{ND}}$, volume, and ${ }^{18} \mathrm{~F}-\mathrm{FDG}$ uptake in ipsilateral (red circles) and contralateral (blue squares) hippocampal ROI. (A) No relationship was observed between epilepsy duration and hippocampal ${ }^{18} \mathrm{~F}-\mathrm{FMZ} \mathrm{BP}_{\mathrm{ND}}$. Significant correlations were found between epilepsy duration and ipsilateral hippocampal volume (B) and normalized ${ }^{18} \mathrm{~F}-\mathrm{FDG}$ uptake $(\mathrm{C})$. 


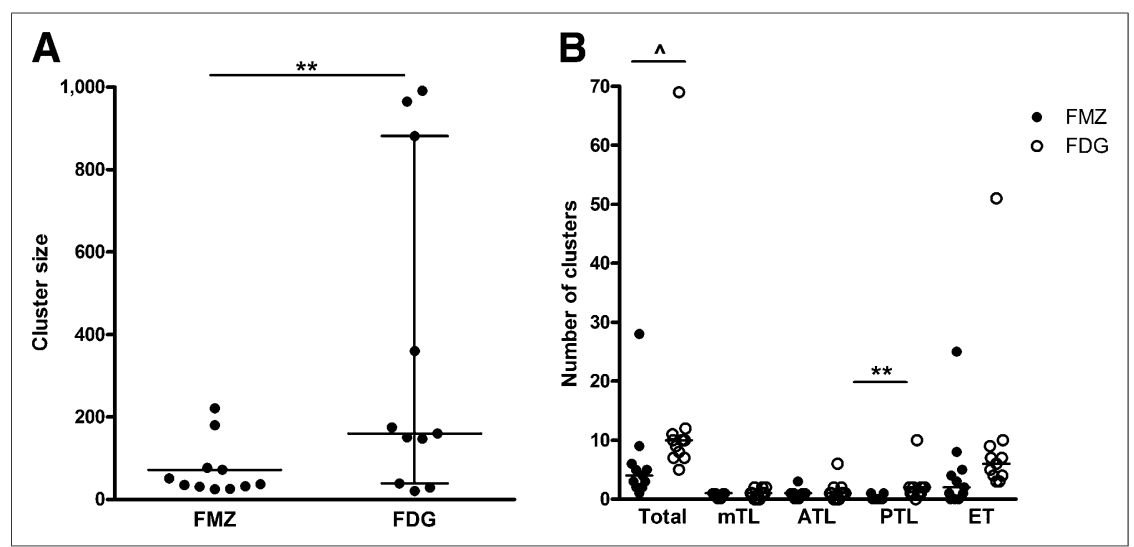

FIGURE 5. (A) Size (no. of voxels) of cluster localizing the epileptogenic zone is shown. ${ }^{18} \mathrm{~F}-$ FMZ cluster size was significantly less than ${ }^{18} \mathrm{~F}-\mathrm{FDG}$ cluster size $(P=0.002)$ when comparing patients who localized on both images $(n=11)$. (B) Number of clusters identified after SPM analysis of both ${ }^{18} \mathrm{~F}-\mathrm{FMZ}$ and ${ }^{18} \mathrm{~F}-\mathrm{FDG}$ images, throughout brain (total); in mesial (mTL), anterior (ATL), and posterior temporal lobes (PTL); and in extratemporal (ET) region are shown. A trend to more clusters on ${ }^{18} \mathrm{~F}-\mathrm{FDG}$ than ${ }^{18} \mathrm{~F}-\mathrm{FMZ}$ was found $(P=0.08)$ and a significantly greater number of clusters identified in the posterior temporal lobe on ${ }^{18} \mathrm{~F}-\mathrm{FDG}$ than ${ }^{18} \mathrm{~F}-\mathrm{FMZ}(P<0.01)$.

lism, or measures of several binding parameters, including $\mathrm{BP}_{\mathrm{ND}}$, between the 2 radiotracers. Importantly, the study found no evidence of specific binding in the pons. Further, Odano et al. showed that $\mathrm{BP}_{\mathrm{ND}}$ values derived from the SRTM were similar to those derived from traditional, invasive compartmental modeling and concluded that ${ }^{18} \mathrm{~F}-\mathrm{FMZ}$ is a suitable alternative to ${ }^{11} \mathrm{C}-\mathrm{FMZ}$ for imaging $\mathrm{GABA}_{\mathrm{A}} / \mathrm{cBZRs}$ in humans (12).

Consistent with previous reports using ${ }^{11} \mathrm{C}-\mathrm{FMZ}$ PET $(7,21)$, we found in this study that ${ }^{18} \mathrm{~F}$-FMZ PET images, whether analyzed visually or using quantitative measures (ROI or SPM), showed a more restricted region of abnormality than did ${ }^{18} \mathrm{~F}-\mathrm{FDG}$ PET images in patients with drug-resistant TLE. ${ }^{18} \mathrm{~F}-\mathrm{FMZ}$ abnormalities tended to be restricted to the hippocampus/mesial temporal region, compared with ${ }^{18} \mathrm{~F}$-FDG hypometabolism, which extended into the surrounding temporal neocortex, agreeing with previous studies with ${ }^{11} \mathrm{C}$-FMZ $(22,23)$.

The visual analysis of the ${ }^{18} \mathrm{~F}-\mathrm{FMZ}$ PET SUV images had a higher localization rate than that for the parametric $\mathrm{BP}_{\mathrm{ND}}$ images. However, the SUV images falsely localized the epileptogenic zone in 3 cases (Table 2), highlighting that localization by nonparametric SUV images should be interpreted with some caution, as has had previously been noted by others $(20,24)$. The visual inspection of both the SUV and the $\mathrm{BP}_{\mathrm{ND}}{ }^{18} \mathrm{~F}-\mathrm{FMZ}$ PET images had a lower localization sensitivity than that for ${ }^{18} \mathrm{~F}-\mathrm{FDG}$ PET images. In contrast, previous studies with ${ }^{11} \mathrm{C}$-FMZ PET have generally reported a higher sensitivity than found in this study. Ryvlin et al. (7) reported that visual inspection of ${ }^{11} \mathrm{C}$-FMZ uptake images resulted in localization of the epileptogenic zone in $94 \%$ of TLE patients, including $100 \%$ of patients with unilateral MTS, $86 \%$ with normal MR imaging findings, and $66 \%$ with bilateral lesions. It is possible that our use of a reference tissue model (SRTM) to generate the parametric $\mathrm{BP}_{\mathrm{ND}}$ images resulted in a lower localization sensitivity than if an arterial plasma input function method had been used (20). A future study that directly compares ${ }^{11} \mathrm{C}-\mathrm{FMZ}$ PET and ${ }^{18} \mathrm{~F}-\mathrm{FMZ}$ PET images in the same patient population, using the same analysis methodology, is required to make definitive conclusions about the relative sensitivity of the 2 tracers for PET imaging in drug-resistant TLE. Of note, although all patients were selected on the basis of ${ }^{18} \mathrm{~F}-\mathrm{FDG}$ hypometabolism being identified on clinical reading, which incorporated correlation with electroencephalography and MR imaging findings, masked reading of the ${ }^{18} \mathrm{~F}-\mathrm{FDG}$ studies yielded a lower sensitivity (72.4\%). Accordingly, ${ }^{18}$ F-FMZ scanning may also perform better in a clinical setting wherein more subtle abnormalities may be considered significant if concordant with other clinical findings.

A previous study investigating the use of SPM in analyzing ${ }^{11} \mathrm{C}-\mathrm{FMZ}$ PET in TLE patients found that SPM localized an ipsilateral decrease in ${ }^{11} \mathrm{C}-\mathrm{FMZ} \mathrm{BP}_{\mathrm{ND}}$ in $87 \%$ of patients. This rate was higher than that found when analyzing static images $(80 \%)$ but lower than the detection rate when using an invasive model (volume of distribution, 100\%) (20). The results of the current study did not attain this level of detection; however, the SPM analysis supported the results of the ROI and visual analyses, indicating that the changes in ${ }^{18} \mathrm{~F}-\mathrm{FMZ} \mathrm{BP}_{\mathrm{ND}}$ are more restricted to the epileptogenic zone than those of hypometabolism on ${ }^{18} \mathrm{~F}$-FDG PET. This was demonstrated by a smaller number and size of abnormal clusters that were focused in the mesial temporal region.

\section{CONCLUSION}

This study investigated the use of ${ }^{18} \mathrm{~F}$-FMZ PET for the localization of the epileptogenic zone in patients with drug-resistant epilepsy. The results agree with previous ${ }^{11} \mathrm{C}-\mathrm{FMZ}$ studies, with decreases in ${ }^{18} \mathrm{~F}-\mathrm{FMZ} \mathrm{BP}_{\mathrm{ND}}$ in the hippocampus, which are more marked on the epileptogenic side. The region of decreased ${ }^{18} \mathrm{~F}-\mathrm{FMZ}$ $\mathrm{BP}_{\mathrm{ND}}$ is more restricted than that of hypometabolism on ${ }^{18} \mathrm{~F}-\mathrm{FDG}$ PET, indicating that it may provide a more specific localization of the epileptogenic zone. Further, the alterations in ${ }^{18} \mathrm{~F}-\mathrm{FMZ} \mathrm{BP}_{\mathrm{ND}}$ were independent of both ${ }^{18} \mathrm{~F}$-FDG uptake and volumes, whereas the latter 2 were correlated, indicating that ${ }^{18} \mathrm{~F}-\mathrm{FMZ}$ may provide a different and complementary measure of the epileptogenic substrate to these established clinical assessments. Importantly, the ${ }^{18} \mathrm{~F}$ $\mathrm{FMZ} \mathrm{BP}_{\mathrm{ND}}$ images could be constructed using a method that did not require arterial blood sampling, making it suitable for routine clinical practice. Overall, the results of this early-phase study support the need for subsequent larger studies to establish the potential of ${ }^{18} \mathrm{~F}-\mathrm{FMZ}$ PET as a clinical tool in the presurgical evaluation of drug-resistant epilepsy-including extratemporal or atypical epilepsies-and to assess its incremental localizing value in comparison with ${ }^{18} \mathrm{~F}$-FDG PET and MR imaging.

\section{DISCLOSURE}

The costs of publication of this article were defrayed in part by the payment of page charges. Therefore, and solely to indicate this fact, this article is hereby marked "advertisement" in accordance with 18 USC section 1734 . This study was funded by the Cooperative Research Centre for Biomedical Imaging Development Ltd. (CRCBID), Bundoora, Victoria, Australia, established and supported under the Australian Government's Cooperative Research Centers program. Core academic partners of the CRC for Biomedical Imaging Development are the Australian Nuclear Science and Technology Organization (ANSTO), Menai, NSW; the Garvan Institute of Med- 
ical Research, Darlinghurst, NSW; Monash University, Clayton, VIC; and the Peter MacCallum Cancer Centre, Melbourne, VIC, all in Australia. Core commercial partners are Berthold Australia Pty Ltd., Bundoora, VIC; Cyclotek (Aust) Pty Ltd., Bundoora, VIC, Australia; and GE Healthcare Pty Ltd., Rydalmere, NSW, Australia. No other potential conflict of interest relevant to this article was reported.

\section{ACKNOWLEDGMENTS}

We thank Sue Belbin and Marian Todaro of the Department of Neurology, Royal Melbourne Hospital, for assistance with patient recruitment. We also thank Jason Callahan and the staff at The Centre for Molecular Imaging, Peter MacCallum Cancer Centre, for assistance with PET scanning.

\section{REFERENCES}

1. Kilpatrick C, O'Brien T, Matkovic Z, Cook M, Kaye A. Preoperative evaluation for temporal lobe surgery. J Clin Neurosci. 2003;10:535-539.

2. Bouilleret V, Valenti MP, Hirsch E, Semah F, Namer IJ. Correlation between PET and SISCOM in temporal lobe epilepsy. J Nucl Med. 2002;43:991-998.

3. Vinton AB, Carne R, Hicks RJ, et al. The extent of resection of FDG-PET hypometabolism relates to outcome of temporal lobectomy. Brain. 2007;130: $548-560$.

4. O'Brien TJ, Miles K, Ware R, Cook MJ, Binns DS, Hicks RJ. The cost-effective use of ${ }^{18} \mathrm{~F}$-FDG PET in the presurgical evaluation of medically refractory focal epilepsy. J Nucl Med. 2008;49:931-937.

5. Hand KS, Baird VH, Van Paesschen W, et al. Central benzodiazepine receptor autoradiography in hippocampal sclerosis. Br J Pharmacol. 1997;122: 358-364.

6. Koepp MJ, Labbe C, Richardson MP, et al. Regional hippocampal $\left[{ }^{11} \mathrm{C}\right]$ flumazenil PET in temporal lobe epilepsy with unilateral and bilateral hippocampal sclerosis. Brain. 1997;120:1865-1876.

7. Ryvlin P, Bouvard S, Le Bars D, et al. Clinical utility of flumazenil-PET versus $\left[{ }^{18} \mathrm{~F}\right]$ fluorodeoxyglucose-PET and MR imaging in refractory partial epilepsy: a prospective study in 100 patients. Brain. 1998;121:2067-2081.

8. Delforge J, Syrota A, Bottlaender M, et al. Modeling analysis of $\left[{ }^{11} \mathrm{C}\right]$ flumazenil kinetics studied by PET: application to a critical study of the equilibrium approaches. J Cereb Blood Flow Metab. 1993;13:454-468.

9. Sanabria-Bohórquez SM, Labar D, Leveque P, et al. $\left[{ }^{11} \mathrm{C}\right]$ flumazenil metabolite measurement in plasma is not necessary for accurate brain benzodiazepine receptor quantification. Eur J Nucl Med. 2000;27:1674-1683.
10. Dedeurwaerdere S, Gregoire MC, Vivash L, et al. In-vivo imaging characteristics of two fluorinated flumazenil radiotracers in the rat. Eur J Nucl Med Mol Imaging. 2009;36:958-965.

11. Gründer G, Siessmeier T, Lange-Asschenfeldt C, et al. $\left[{ }^{18} \mathrm{~F}\right]$ fluoroethylflumazenil: a novel tracer for PET imaging of human benzodiazepine receptors. Eur $J$ Nucl Med. 2001;28:1463-1470.

12. Odano I, Halldin C, Karlsson P, et al. $\left[{ }^{18} \mathrm{~F}\right]$ flumazenil binding to central benzodiazepine receptor studies by PET: quantitative analysis and comparisons with [ $\left.{ }^{11} \mathrm{C}\right]$ flumazenil. Neuroimage. 2009;45:891-902.

13. Ryzhikov NN, Seneca N, Krasikova RN, et al. Preparation of highly specific radioactivity $\left[{ }^{18} \mathrm{~F}\right]$ flumazenil and its evaluation in cynomolgus monkey by positron emission tomography. Nucl Med Biol. 2005;32:109-116.

14. Gilliam F, Faught E, Martin R, et al. Predictive value of MR imaging-identified mesial temporal sclerosis for surgical outcome in temporal lobe epilepsy: an intent-to-treat analysis. Epilepsia. 2000;41:963-966.

15. Gunn RN, Lammertsma AA, Hume SP, Cunningham VJ. Parametric imaging of ligand-receptor binding in PET using a simplified reference region model. Neuroimage. 1997;6:279-287.

16. Bouvard S, Costes N, Bonnefoi F, et al. Seizure-related short-term plasticity of benzodiazepine receptors in partial epilepsy: a $\left[{ }^{11} \mathrm{C}\right]$ flumazenil-PET study. Brain. 2005; 128:1330-1343

17. Babb JL, Pretorius JK. Pathological substrates of epilepsy. In: Wyllie E, ed. The Treatment of Epilepsy: Principles and Practice. Philadelphia, PA: Lippincott, Williams \& Wilkins; 1993:55-70.

18. Koepp MJ, Richardson MP, Labbe C, et al. ${ }^{11} \mathrm{C}$-flumazenil PET, volumetric MR imaging, and quantitative pathology in mesial temporal lobe epilepsy. Neurology. 1997;49:764-773.

19. Szelies B, Weber-Luxenburger G, Mielke R, et al. Interictal hippocampal benzodiazepine receptors in temporal lobe epilepsy: comparison with coregistered hippocampal metabolism and volumetry. Eur J Neurol. 2000;7:393-400.

20. Hammers A, Panagoda P, Heckemann RA, et al. $\left[{ }^{11} \mathrm{C}\right]$ flumazenil PET in temporal lobe epilepsy: do we need an arterial input function or kinetic modeling? J Cereb Blood Flow Metab. 2008;28:207-216.

21. Padma MV, Simkins R, White $\mathrm{P}$, et al. Clinical utility of ${ }^{11} \mathrm{C}$-flumazenil positron emission tomography in intractable temporal lobe epilepsy. Neurol India. 2004; 52:457-462.

22. Henry TR, Frey KA, Sackellares JC, et al. In vivo cerebral metabolism and central benzodiazepine-receptor binding in temporal lobe epilepsy. Neurology. 1993;43:1998-2006.

23. Savic I, Thorell JO, Roland P. $\left[{ }^{11} \mathrm{C}\right]$ flumazenil positron emission tomography visualizes frontal epileptogenic regions. Epilepsia. 1995;36:1225-1232.

24. Ryvlin P, Bouvard S, Le Bars D, Mauguiere F. Transient and falsely lateralizing flumazenil-PET asymmetries in temporal lobe epilepsy. Neurology. 1999;53: 1882-1885. 